\title{
Dynamics of charge separation at an organic donor-acceptor interface
}

\author{
Zhen Sun and Sven Stafström
}

\section{Linköping University Post Print}

\section{Tweet}

N.B.: When citing this work, cite the original article.

Original Publication:

Zhen Sun and Sven Stafström, Dynamics of charge separation at an organic donor-acceptor interface, 2014, Physical Review B. Condensed Matter and Materials Physics, (90), 11, 115420. http://dx.doi.org/10.1103/PhysRevB.90.115420

Copyright: American Physical Society

http://www.aps.org/

Postprint available at: Linköping University Electronic Press

http://urn.kb.se/resolve?urn=urn:nbn:se:liu:diva-111451 


\title{
Dynamics of charge separation at an organic donor-acceptor interface
}

\author{
Zhen Sun* and Sven Stafström ${ }^{\dagger}$ \\ Department of Physics, Chemistry, and Biology, Linköping University, SE-58183 Linköping, Sweden \\ (Received 5 December 2013; revised manuscript received 15 August 2014; published 15 September 2014)
}

\begin{abstract}
The key process in organic photovoltaic cells is the charge separation at organic donor-acceptor interfaces. However, exactly how the charges separate into free charge carriers still remains a puzzle. We present here simulations of the electron dynamics of this process using a nonadiabatic Ehrenfest method. From these simulations, we give a direct illustration of the charge separation process. The results show that the delocalization of the electronic states involved plays a critical role in order to overcome the Coulomb attraction of the charge transfer (CT) exciton. Charge separation only occurs for sufficiently strong intermolecular interactions. Alternatively, the CT exciton relaxes into a bound polaron pair. The results also show that the "excess energy" of the hot CT exciton facilitates the charge separation process to a certain degree.
\end{abstract}

DOI: 10.1103/PhysRevB.90.115420

PACS number(s): 88.40.jr, 71.38.-k, 72.20.Jv, 72.80.Le

The process of generation of free charge carriers in organic photovoltaic cells (OPVs) based on donor-acceptor (D/A) materials is rather complex with the most critical steps occurring at the D/A interface. First, photon absorption leads to exciton formation in the donor material. In order for the exciton to dissociate it has to diffuse towards the D/A interface. Once the exciton reaches the D/A interface, the excited electron can be transferred to the acceptor if the energy offset of the donor and acceptor is sufficient to overcome the exciton binding energy. The presently accepted description is that an interfacial charge transfer (CT) exciton is formed, i.e., a Coulombically bound electron-hole pair located on adjacent donor and acceptor molecules.

The binding energy of the interfacial CT exciton is approximately an order of magnitude higher than thermal energy at room temperature [1]. Even so, the efficiency of free charge carrier generation is close to unity in optimized polymer OPVs. How the interfacial CT excitons can overcome such a high binding energy to produce photocurrent has been extensively discussed but up to now there is no unified picture of how this occurs. A hot CT exciton mechanism was proposed to explain the high efficiency of charge separation in OPVs [2-5]. The hot CT exciton means an excited CT exciton with "excess energy" inherited from the exciton. It is assumed that this "excess energy" would not dissipate into heat; instead it is used to promote the dissociation into free charges. However, experiments have shown that the charge transfer can be obtained directly from the ground state by illumination of photons well below the optical gap of the donor; i.e., the "excess energy" is not necessary for the CT exciton dissociation [6,7].

Another possible explanation is related to electrical screening. As is well known, the microelectrostatic environment at the organic D/A interface is significantly different from that in bulk. Dielectric constant, molecular packing structure, and molecular multipole moments will vary near the interface. It has been argued that this results in a strong effective screening and would therefore facilitate charge separation at the D/A interface [8-13]. A third explanation which has

\footnotetext{
*zhesu@ifm.liu.se

†svens@ifm.liu.se
}

been discussed in the context of charge separation is related to the character of the electronic states involved. If these states are delocalized, the charge separation process is greatly facilitated [4,14-16] and the effect of the "excess energy" would be marginal. Our simulations show results that are in agreement with this explanation. In particular, we show that the CT exciton state hardly exists if the electron is transferred to a delocalized state in the donor system. In this case there is practically instant separation of the charges into free carriers. Such an observation was made possible via detailed dynamical simulations following the electron dynamics as well as the lattice dynamics. These simulations are based on the nonadiabatic Ehrenfest dynamics [17]. Previously, we have studied exciton dissociation in a system composed of a donor and an acceptor polymer chain [18]. In this work, we extend this study to include also the most critical step for photovoltaics, the charge separation for which we need to perform simulations on a considerably larger system of polymer chains, which can hold two fully separated charges. These chains are placed in configuration $\mathrm{D}_{1} \ldots \mathrm{D}_{7} \mathrm{~A}_{1} \ldots \mathrm{A}_{7}$, as shown in Fig. 1.

The total Hamiltonian of the D/A system can be expressed as

$$
\hat{H}=\hat{H}_{\mathrm{el}}+\hat{H}_{\mathrm{ee}}+\hat{H}_{\mathrm{latt}} \cdot
$$

The first term is the single-electron Hamiltonian which includes the electron-lattice coupling

$$
\hat{H}_{\mathrm{el}}=\sum_{i, s} \Delta_{i} \hat{c}_{i, s}^{\dagger} \hat{c}_{i, s}-\sum_{i, j, s} t_{i, j}\left(\hat{c}_{i, s}^{\dagger} \hat{c}_{j, s}+\hat{c}_{j, s}^{\dagger} \hat{c}_{i, s}\right),
$$

where $i$ runs over the sites of all the donor and acceptor chains (a simplified notation is used without a chain index on $i$ ). $\Delta_{i}$ denotes the on-site energy of site $i$. To mimic the D/A blend system, we set different values of the on-site energy on the donor chains and acceptor chains, $\Delta_{i}=\Delta E$ for all sites of the donor chains and $\Delta_{i}=0$ for all sites of acceptor chains. The hopping integral between two sites $i$ and $j$ is denoted by $t_{i, j}$. This quantity includes both intra- and interchain hopping, where the former includes the electron-lattice coupling, in the same way as described earlier [18]. The interchain hopping integrals are denoted by $t_{\mathrm{v}}$ and $t_{\mathrm{DA}} . t_{\mathrm{v}}$ is the interchain hopping between two neighboring donor (acceptor) chains, while $t_{\mathrm{DA}}$ is 


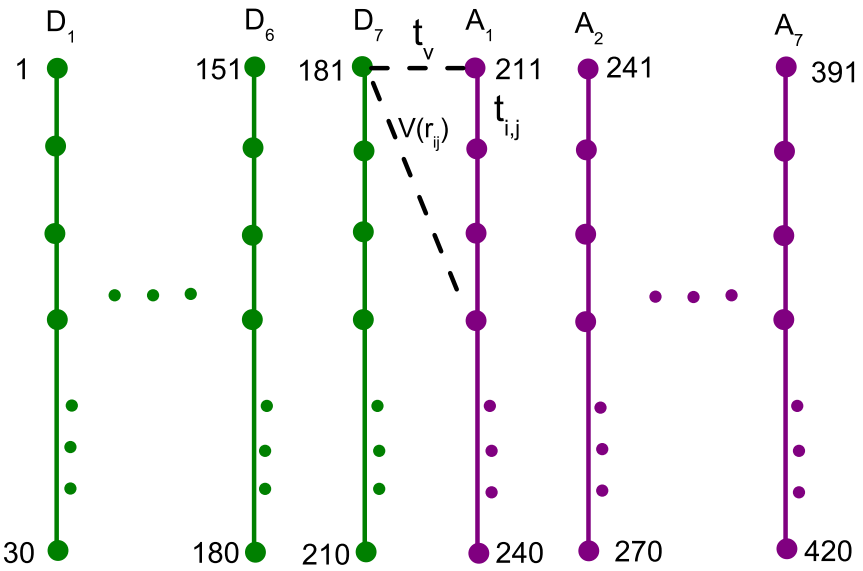

FIG. 1. (Color online) Schematic diagram of the D-A blend system.

the interchain hopping between the donor and acceptor chains that form the D/A interface (chains $\mathrm{D}_{7}$ and $\mathrm{A}_{1}$; see Fig. 1). Here, $t_{\mathrm{DA}}$ is described in relation to $t_{\mathrm{v}}$,

$$
t_{\mathrm{DA}}=t_{\mathrm{v}} \exp \left(1-\frac{d_{\mathrm{DA}}}{d}\right),
$$

where $d_{\mathrm{DA}}$ and $d$ are the interchain distance between $\mathrm{D}_{7}$ and $A_{1}$ and between two neighboring donor (acceptor) chains, respectively.

We also perform calculations including disorder in the on-site energies as well as in the hopping integrals. In both these quantities we make use of an uncorrelated set of random numbers drawn from a Gaussian distributions. The effect of this type of disorder on the charge separation process is discussed briefly below.

The second contribution in Eq. (1) describes the electronelectron interactions,

$$
\begin{aligned}
\hat{H}_{\mathrm{ee}}= & U \sum_{i}\left(\hat{n}_{i \uparrow}-\frac{1}{2}\right)\left(\hat{n}_{i \downarrow}-\frac{1}{2}\right) \\
& +\frac{1}{2} \sum_{i, j \neq i} V\left(r_{i j}\right)\left(\hat{n}_{i}-1\right)\left(\hat{n}_{j}-1\right),
\end{aligned}
$$

where $U$ denotes the screened on-site Coulomb interactions, $\hat{n}_{i, s}=\hat{c}_{i, s}^{\dagger} \hat{c}_{i, s}, \hat{n}_{i}=\sum_{s} \hat{c}_{i, s}^{\dagger} \hat{c}_{i, s}$, and $V\left(r_{i j}\right)$ is the Ohno potential defined as

$$
V\left(r_{i j}\right)=\frac{U}{\sqrt{1+\left(\beta r_{i j} / r_{0}\right)^{2}}} .
$$

The Ohno potential describes the long-range Coulomb interactions, where $r_{i j}$ denotes the distance between sites $i$ and $j$, $r_{0}$ the average bond length, and $\beta$ the screening factor.

The last term in Eq. (1) is the Hamiltonian of the lattice backbone which is treated classically,

$$
\hat{H}_{\text {latt }}=\frac{K}{2} \sum_{i}\left(u_{i+1}-u_{i}\right)^{2}+\frac{M}{2} \sum_{i} \dot{u}_{i}^{2},
$$

where $u_{i}$ is the monomer displacement of site $i, K$ is the elastic constant of a $\sigma$ bond, and $M$ the mass of a $\mathrm{CH}$ group.

The various parameters used in the calculations are chosen so as to model cis-polyacetylene. The system can, however, be considered as a model system which is able to grasp the essentials of most (nondegenerate) conjugated polymers. We adopt the parameters developed by Miranda et al. [19,20], which also were used in our previous work [18,21,22]. Specific attention is given to three parameters: the interchain hopping $t_{\mathrm{v}}$ and $t_{\mathrm{DA}}$, the screening of the Coulomb potential $\beta$, and the band offset $\Delta E$. Three sets of simulations are presented below; in each such simulation we vary one of these parameters and keep the other two at a fixed value.

Within the nonadiabatic electron-lattice dynamics method [17], the electrons are treated quantum mechanically and evolve according to the time-dependent Schrödinger equation. The atoms, on the other hand, are considered as classical particles, governed by Newton's equations of motion, and move under the effects of nuclear and electronic contributions. We use a multiconfigurational time-dependent Hartree-Fock (MCTDHF) method introduced in Refs. [19,20] to solve the time-dependent Schrödinger equation. The evolution of the system is obtained by time integration of the two sets of equations of motion. The numerical methods have been described previously [18,21].

For the simulations discussed below, we use the mean charge density $\bar{\rho}_{i}(t)$ to analyze and display the charge density evolution,

$$
\bar{\rho}_{i}(t)=\frac{1}{4}\left[\rho_{i-1, i-1}(t)+2 \rho_{i, i}(t)+\rho_{i+1, i+1}(t)\right],
$$

where the charge density $\rho_{i, j}(t)=\sum_{s}\left\langle\hat{c}_{i, s}^{\dagger} \hat{c}_{j, s}\right\rangle$. In addition to the charge density, which is obtained from the time-dependent Schrödinger equation, the solution to Newton's equations of motion for the site displacements $u_{i}$ is also of interest and will be discussed (see also Supplemental Material (SM) [23]) in connection to the results showing the mean charge densities.

A set of simulations was carried out for different interchain interactions with screening of the Coulomb potential $\beta=$ 3.4 [20] and band offset $\Delta E=0.5 \mathrm{eV}$. In order to reflect the differences of the interchain interactions at the interface and at other places, we set $d_{\mathrm{DA}}=6 \AA$ and $d=5 \AA$. According to Eq. (3) and Eq. (5), this means that the interchain interactions including interchain electron-lattice interactions and interchain electron-electron interactions at the interface are slightly weaker than other places. The resulting time-dependent charge densities [see Eq. (7)] are shown in Fig. 2.

The initial state is $D_{1} \ldots D_{7}^{*} A_{1} \ldots A_{7}$, which means the exciton resides on the seventh donor chain while other chains are in the ground state. In the case of weak interchain interactions [see Fig. 2(a)], in which the interchain hopping is set to $30 \mathrm{meV}$, the exciton is initially localizes to a single chain, the donor chain $\mathrm{D}_{7}$, whose site number is from 181 to 210 . Since the exciton is neutral we cannot see it on the figures of charge density evolution. It is clearly seen, however, in the lattice displacements obtained from Eq. (6) (see SM [23]).

In order to avoid spurious effects, the interchain interactions are gradually turned on over the first 75 fs of the simulations. Therefore, from 0 to about $100 \mathrm{fs}$, the charge density is zero all over the donor and acceptor chains because the interchain interactions are not large enough to make the exciton dissociate. From about $100 \mathrm{fs}$, we can see that there are positive and negative charges appearing on $\mathrm{D}_{7}$ and $\mathrm{A}_{1}$, respectively. Obviously, this means that the exciton starts to dissociate. From 

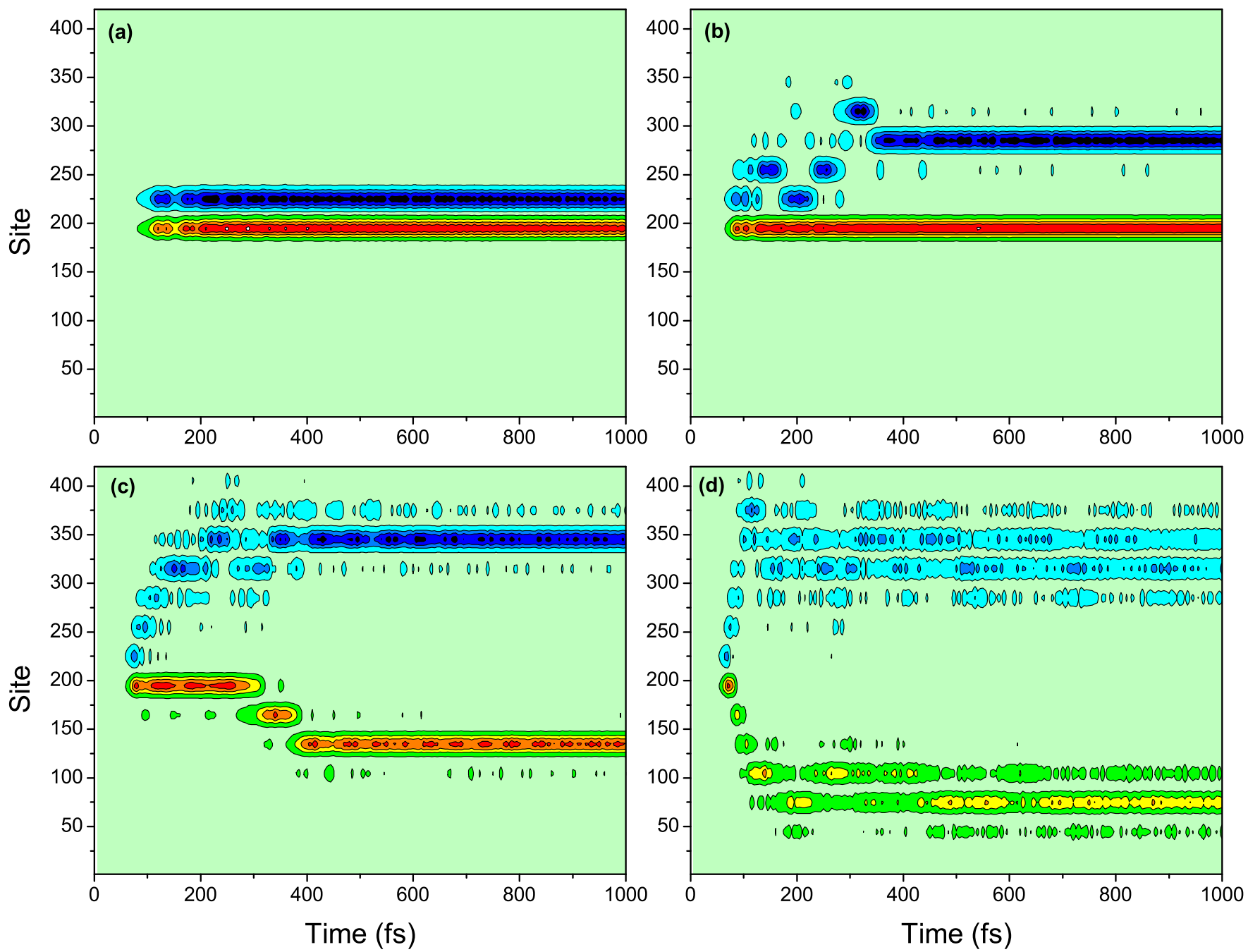

FIG. 2. (Color online) Time evolution of the mean charge density $\bar{\rho}_{i}(t)$ with different interchain interactions: (a) $t_{\mathrm{v}}=30 \mathrm{meV}$, (b) $t_{\mathrm{v}}=$ $50 \mathrm{meV}$, (c) $t_{\mathrm{v}}=70 \mathrm{meV}$, and (d) $t_{\mathrm{v}}=100 \mathrm{meV}, d_{\mathrm{DA}}=6 \AA, d=5 \AA, \beta=3.4$, and $\Delta E=0.5 \mathrm{eV}$, and $t_{\mathrm{DA}}$ is derived from Eq. (3).

$100 \mathrm{fs}$ to $200 \mathrm{fs}$, these positive and negative charges undergo some oscillations but from 200 fs and onwards we observe a steady-state solution with unit net charges on chains $\mathrm{D}_{7}$ and $\mathrm{A}_{1}$ : $\mathrm{D}_{1} \ldots \mathrm{D}_{7}^{+} \mathrm{A}_{1}^{-} \ldots \mathrm{A}_{7}$. If we combine this charge density evolution with lattice evolution (see SM [23]), we conclude that charges on $\mathrm{D}_{7}$ and $\mathrm{A}_{1}$ correspond to positive and negative polarons, which are tightly bound together by the attractive Coulomb force that acts between these localized charge distributions.

In the next panel, Fig. 2(b), for which the interchain hopping is $50 \mathrm{meV}$, the exciton starts to dissociate at about 75 fs. By studying the evolution of the atomic displacements (see SM [23]), it is seen that the hole remains in the same geometrical defect as the exciton; i.e., a positive polaron is formed in the donor system. Due to the fact that the hole occupies a state which is localized as a consequence of the initial condition, namely, the existence of a localized exciton on $\mathrm{D}_{7}$, the positive polaron is essentially immobile for the chosen strength of the interchain hopping. The electron which is transferred to the acceptor system is, however, transferred to an electronic state which is not associated with a geometrical defect and which therefore has a much larger tendency for delocalization than the hole state. This explains why the negative polaron hops back and forth among the acceptor chains, and, as the state cools down, eventually localizes on $\mathrm{A}_{3}$. The final state is $\mathrm{D}_{1} \ldots \mathrm{D}_{7}^{+} \mathrm{A}_{1} \mathrm{~A}_{2} \mathrm{~A}_{3}^{-} \ldots \mathrm{A}_{7}$. Since the distance between the two polarons is not far, we can call this state a weakly bound polaron pair.

When the interchain hopping strength is increased to $70 \mathrm{meV}$, the acceptor state is even more delocalized and the transferred electron is less bound to the D/A interfaces. In this case, fully separated positive and negative polarons are obtained in the final state of the simulation: $\mathrm{D}_{1} \ldots \mathrm{D}_{5}^{+} \mathrm{D}_{6} \mathrm{D}_{7} \mathrm{~A}_{1} \ldots \mathrm{A}_{5}^{-} \mathrm{A}_{6} \mathrm{~A}_{7}$. It should be noted that the ground state of a polaron in either the donor or the acceptor parts of the system at this strength of interchain interactions corresponds to a polaron in the central region of each system, i.e., very close to the pattern observed in Fig. 2(c). The Coulomb attraction between the negative polaron and the positive polaron is very weak due to the large distance between them.

As was noted above, the asymmetry between the donor and acceptor parts is due to the fact that initially there is a geometrical deformation of the donor chain associated with the exciton. The time interval over which the electron moves away from the D/A interface is extremely short. This process is 

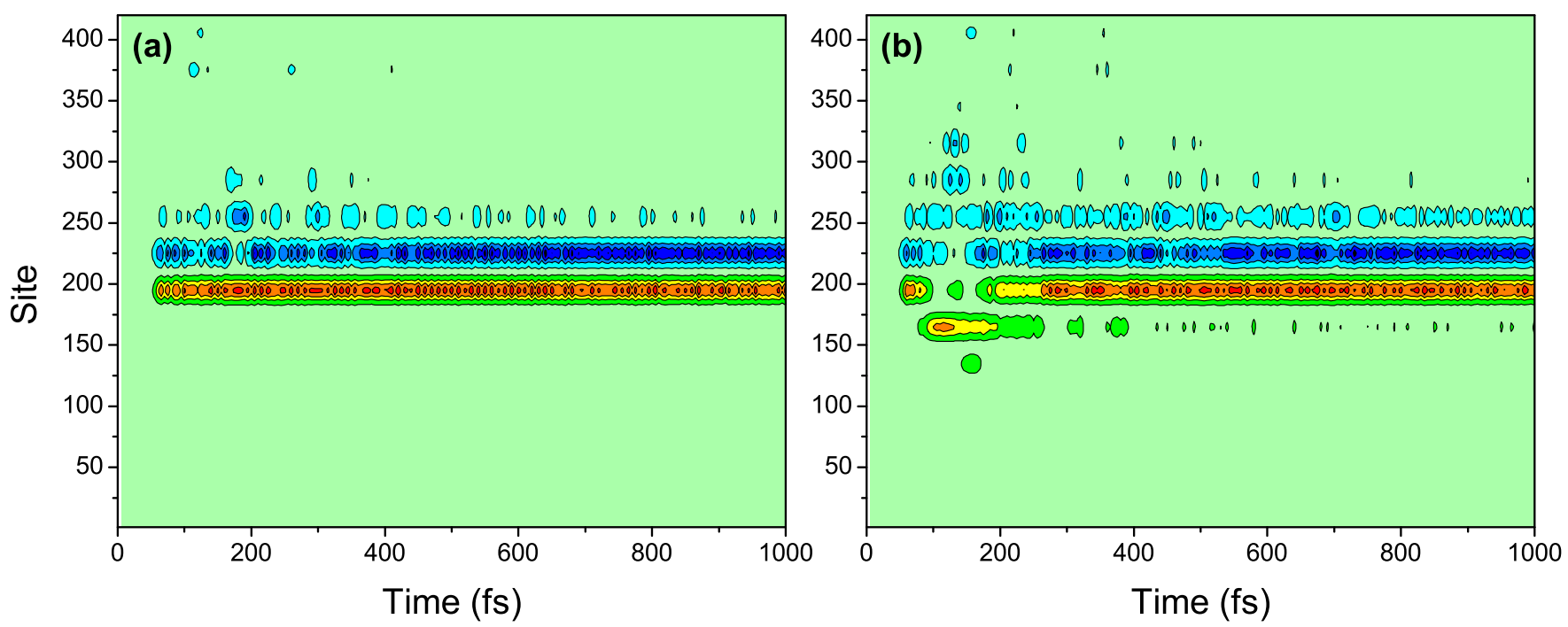

FIG. 3. (Color online) Panels (a) and (b) are the same simulations as Figs. 2(c) and 2(d), respectively, but with a shorter interchain distance at the interface, $d_{\mathrm{DA}}=5 \AA$.

made possible due to the delocalized nature of acceptor states. It is worth stressing that the fact that the electron is transferred to a high-lying unoccupied level in the acceptor system is of less importance in this context; it is the delocalized nature of the acceptor state which gives rise to charge separation. The strength of the interchain interactions also allows the hole polaron to move between chains. The driving force for this motion is related to the fact that the ground state of the donor part of the system corresponds to a polaron located in the middle of this system.

At even larger interchain interactions, for $t_{\mathrm{v}}=100 \mathrm{meV}$, we obtain essentially free charge carriers as shown in Fig. 2(d). The behavior follows the trend displayed in Figs. 2(a)2(c); increasing interchain interactions leads to interchain delocalization and charge separation. Due to the strong interchain interactions, the negative and positive charges are both delocalized over several chains in this case. The geometrical distortions associated with these charges are very small and consequently, the polarons formed have very low binding energies and are essentially band states.

We note that in the simulations presented above, the interchain distance at the D/A interface is always larger than the interchain distance within the donor (acceptor) subsystems. We have carried out the same set of simulations with the two interchain distances (interactions) set to the same value: $d_{\mathrm{DA}}=d=5 \AA$ [see also Eq. (3)]. In particular, for the values of $t_{\mathrm{v}}$ presented in Figs. 2(c) and 2(d) we now obtain the time evolution of charge densities shown in Figs. 3(a) and 3(b), respectively. Evidently, with $d_{\mathrm{DA}}$ decreased to the same value as $d$, charge separation no longer happens, even for interchain interactions as high as $100 \mathrm{meV}$. The smaller value we use for the donor-acceptor distances, the larger the Coulomb attraction between the electron and the hole. If the driving force for charge separation caused by wave function delocalization (or $t_{\mathrm{v}}$ ) cannot overcome the Coulomb attraction, charge separation will not occur.

In the following, we performed a set of simulations to investigate the role of Coulomb interactions in the charge separation process by changing the value of the screening factor $\beta$ in Eq. (5). In Fig. 4, we show two simulations which are performed the same as in Fig. 2(a) but with different screening factors: (a) $\beta=4.0$ and (b) $\beta=4.4$. When the screening factor is increased [from the value of $\beta=3.4$ used in the simulations presented in Fig. 2(a)] there is a corresponding decrease in the Coulomb attraction between the two polarons with opposite charges. Therefore, charge separation should change to be easier. As expected, from panels (a) and (b) in Fig. 4 we can see that the two polarons are separated, and the larger the screening factor, the farther the two polarons separate. The separation process shown in Fig. 4 is similar to that displayed in Fig. 2(b), in which the motion of the polaron between the chains is of hopping type. Thus, for weak enough Coulomb attraction, it is possible even for a pair of localized polarons to separate from each other. It should be noted that we have not performed investigations on the relevance of the values of the screening. The standard Ohno value of $\beta=3.4$ is used for bulk materials and since interface charges have been shown to increase screening [8-13] it is likely that the value of $\beta$ is larger than the value used by Miranda [20]. Furthermore, depending on the structure at the interface, there is most likely a varying strength of the screening which might result in different tendencies for charge separation at different positions along the interface.

The critical role of delocalization of the wave function is further tested here by including disorder into the model. We introduce static disorder by including two uncorrelated sets of Gaussian distributed random numbers on the on-site energy $\Delta_{i}$ and interchain hopping integral $t_{\mathrm{v}}$, respectively. The mean of each set of random numbers is set to zero. By studying the charge separation process for increasing standard deviations of the distributions we observe a reduction in the charge separation. For example, a standard deviation of $50 \mathrm{meV}$ in the distribution of the on-site energy and $7 \mathrm{meV}$ in the distribution of the interchain hopping integral for the case of $t_{\mathrm{v}}=70 \mathrm{meV}$ [Fig. 2(c)] results in a situation similar to that shown in Fig. 2(b). 

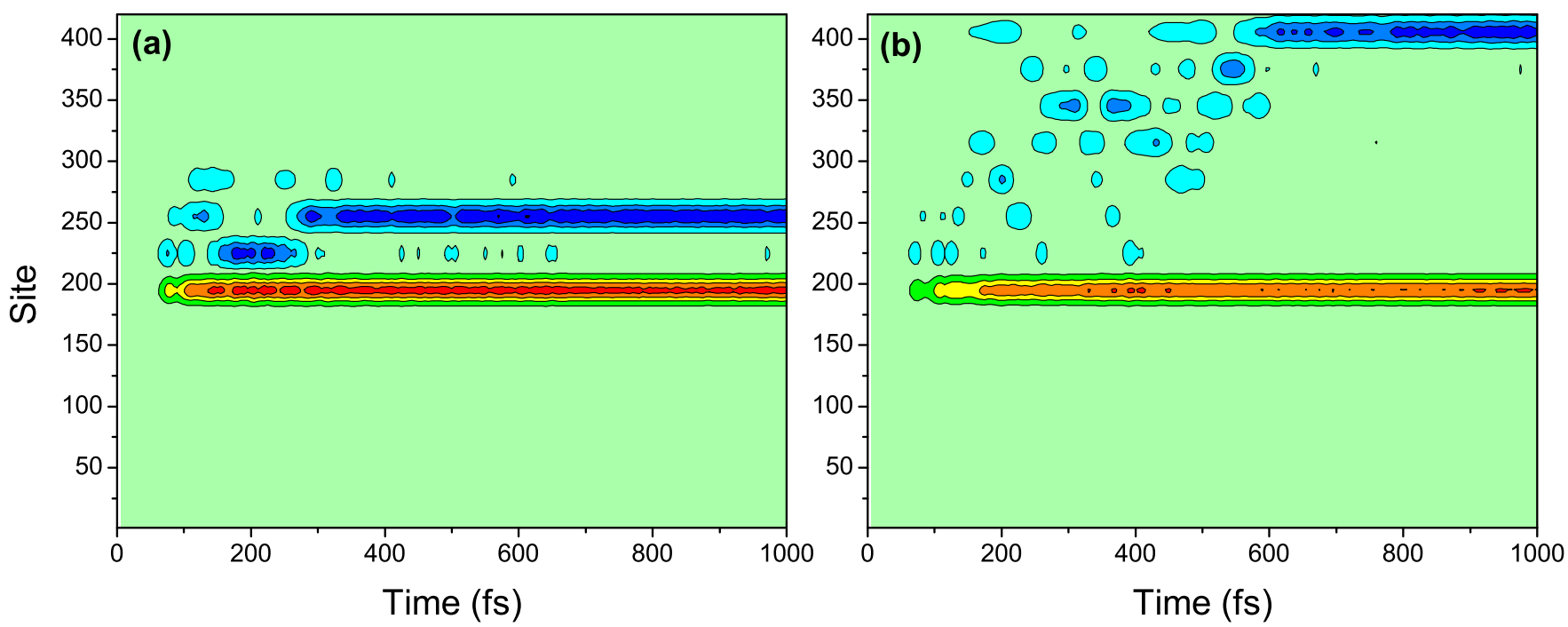

FIG. 4. (Color online) Panels (a) and (b) are the same simulation as Fig. 2(a) but with different screening factors: (a) $\beta=4.0$ and (b) $\beta=4.4$.

The role of the "excess energy" in the charge separation process was introduced above. Here we are able to perform direct simulations that can give a conclusive description of the role of the "excess energy." This is done by varying the band offset $\Delta E$. Larger band offset means more "excess energy." In Fig. 5 is shown the time evolution of the mean charge density for two different band offsets $\Delta E=0.6$ and $\Delta E=0.7$, while other parameters are the same as in Fig. 2(a). Figure 5(a) corresponds to the band offset $\Delta E=0.6$, i.e., 0.1 eV larger than in Fig. 2(a). We see that the exciton begins to dissociate at about $75 \mathrm{fs}$. Due to the additional energy as compared to Fig. 2(a), the negative polaron has a tendency to escape from the D/A interface. However, due to weak interchain interactions, the polaron remains localized, and due to the strong Coulomb attraction, the negative polaron is pulled back to the D/A interface again. The system then

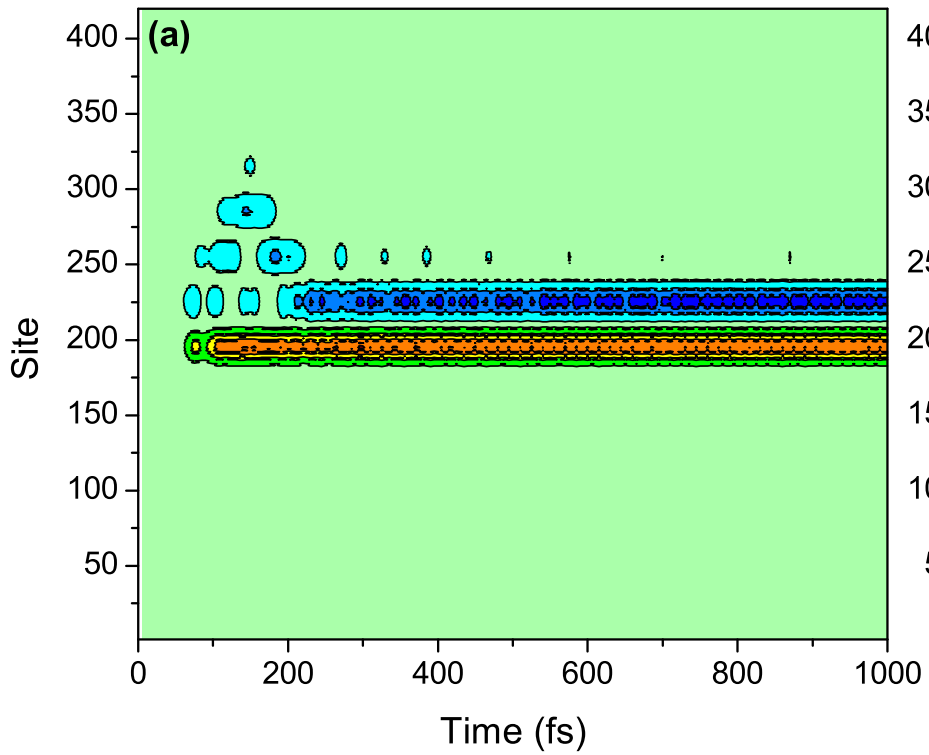

relaxes to the state corresponding to a bound polaron pair, as in Fig. 2(a).

Figure 5(b) corresponds to the band offset $\Delta E=0.7$, i.e., $0.2 \mathrm{eV}$ larger than in Fig. 2(a). Before $400 \mathrm{fs}$, the electron which is transferred to the acceptor system is apparently very delocalized, and distributes nearly every acceptor chains. Obviously, the transferred electron with more "excess energy" cannot be trapped by the positive charge. After $400 \mathrm{fs}$, this delocalized charge finally relaxes and forms a negative polaron localized on $\mathrm{A}_{7}$. Thus, we obtain almost fully separated negative and positive polarons. Compared with Fig. 2(a), we can conclude that more "excess energy" leads to easier charge separation.

From the results shown in Fig. 5, it can be seen that the "excess energy" facilitates charge separation to a certain extent. We would like to emphasis that the comparison made between Figs. 2 and 5 is not relevant for the kind of experiments

FIG. 5. (Color online) Panels (a) and (b) are the same simulations as Fig. 2(a), but with different band offset, (a) $\Delta E=0.6 \mathrm{eV}$ and (b) $\Delta E=0.7 \mathrm{eV}$. 
performed by, e.g., van der Hofstad et al. [7]. In this experiment there is no initial exciton state and both the hole and electron states could in principle be delocalized. In our simulations, as well as in the solar cell device, the exciton which is created in the donor system is localized even in the case of high interchain hopping (see SM [23]). When this exciton dissociates, the hole state is localized and acts as a Coulomb trap for the transferred electron. We have shown here that the "excess energy" can assist in releasing the electron from this trap.

We should note that the Ehrenfest method used in our simulations has some limitations in accuracy. First, it is a mean-field approach in which the phenomenon of energy transfer from electron to lattice [24] is underestimated. As a consequence of this drawback, the electron temperature is to some extent uncontrolled and there is a built-in tendency of delocalization of the electronic wave functions, which can lead to destabilization of the polaron. According to the time evolution of occupation number in our simulations and considering that the charge separation occurs very fast, typically within $100 \mathrm{fs}$, the effect of this built-in tendency of delocalization is therefore very weak and does not significantly affect our results.

A second drawback of the Ehrenfest method is that the lattice degrees of freedom are treated classically. This means that quantum effects such as tunneling and zero-point motion are totally neglected. It is hard to estimate the importance of such effect for the type of simulations presented here but at the same time it is not possible to perform more detailed simulations without reducing the system to a size which makes it irrelevant to study the charge separation process. We believe that the limitations in size of our model, including the number of donor and acceptor chains and the chain length, has some minor effects on the charge separation process, but the qualitative trends observed in this paper are not affected.

To summarize, by using the nonadiabatic Ehrenfest dynamics method, we perform simulations on the process of charge separation in a D/A system. We found that the delocalization of the wave functions on the acceptor side is crucial in order to obtain charge separation. On the one hand we found that this driving force for charge separation dominates over the Coulomb attraction for sufficiently strong interchain interactions. On the other hand, if the interchain distance at the D/A interface is smaller or equal to the interchain distance within the acceptor subsystem, charge separation will not occur even for strong interchain interactions due to the fact that the Coulomb attraction between the electron and the hole will dominate over the driving force for charge separation. As expected, also the strength of the Coulomb interactions plays an important role; at weak such interactions it is possible for a localized electron polaron to escape from the D/A interface. The "excess energy" facilitates the process from the hot CT state to charge separation state to a certain extent.

Financial support from the Vetenskapsrådet and from the Energimyndigheten is gratefully acknowledged.
[1] M. Muntwiler, Q. X. Yang, W. A. Tisdale, and X. Y. Zhu, Phys. Rev. Lett. 101, 196403 (2008).

[2] X.-Y. Zhu, Q. Yang, and M. Muntwiler, Acc. Chem. Res. 42, 1779 (2009).

[3] D. H. K. Murthy, M. Gao, M. J. W. Vermeulen, L. D. A. Siebbeles, and T. J. Savenije, J. Phys. Chem. C 116, 9214 (2012).

[4] A. E. Jailaubekov, A. P. Willard, J. R. Tritsch, W.-L. Chan, N. Sai, R. Gearba, L. G. Kaake, K. J. Williams, K. Leung, P. J. Rossky, and X.-Y. Zhu, Nat. Mater. 12, 66 (2013).

[5] G. Grancini, M. Maiuri, D. Fazzi, A. Petrozza, H-J. Egelhaaf, D. Brida, G. Cerullo, and G. Lanzani, Nat. Mater. 12, 29 (2013).

[6] J. Lee, K. Vandewal, S. R. Yost, M. E. Bahlke, L. Goris, M. A. Baldo, J. V. Manca, and T. V. Voorhis, J. Am. Chem. Soc. 132, 11878 (2010).

[7] T. G. J. van der Hofstad, D. D. Nuzzo, M. van den Berg, R. A. J. Janssen, and S. C. J. Meskers, Adv. Energy Mater. 2, 1095 (2012).

[8] G. D’Avino, S. Mothy, L. Muccioli, C. Zannoni, L. Wang, J. Cornil, D. Beljonne, and F. Castet, J. Phys. Chem. C 117, 12981 (2013).

[9] S. R. Yost and T. V. Voorhis, J. Phys. Chem. C 117, 5617 (2013).

[10] S. Mothy, M. Guillaume, J. Idé, F. Castet, L. Ducasse, J. Cornil, and D. Beljonne, J. Phys. Chem. Lett. 3, 2374 (2012).

[11] S. R. Yost, L.-P. Wang, and T. V. Voorhis, J. Phys. Chem. C 115, 14431 (2011).
[12] D. P. McMahon, D. L. Cheung, and A. Troisi, J. Phys. Chem. Lett. 2, 2737 (2011).

[13] V. I. Arkhipov, P. Heremans, and H. Bässler, Appl. Phys. Lett. 82, 4605 (2003).

[14] A. A. Bakulin, A. Rao, V. G. Pavelyev, P. H. M. van Loosdrecht, M. S. Pshenichnikov, D. Niedzialek, J. Cornil, D. Beljonne, and R. H. Friend, Science 335, 1340 (2012).

[15] A. Troisi, Faraday Discuss. 163, 377 (2013).

[16] P. K. Nayak, K. L. Narasimhan, and D. Cahen, J. Phys. Chem. Lett. 4, 1707 (2013).

[17] S. Stafström, Chem. Soc. Rev. 39, 2484 (2010).

[18] Z. Sun and S. Stafström, J. Chem. Phys. 138, 164905 (2013).

[19] R. P. Miranda, A. J. Fisher, L. Stella, and A. P. Horsfield, J. Chem. Phys. 134, 244101 (2011).

[20] R. P. Miranda, A. J. Fisher, L. Stella, and A. P. Horsfield, J. Chem. Phys. 134, 244102 (2011).

[21] Z. Sun and S. Stafström, J. Chem. Phys. 136, 244901 (2012).

[22] Y. L. Zhang, X. J. Liu, Z. Sun, and Z. An, J. Chem. Phys. 138, 174906 (2013).

[23] See Supplemental Material at http://link.aps.org/supplemental/ 10.1103/PhysRevB.90.115420 the motion of the site displacements.

[24] A. P. Horsfield, D. R. Bowler, H. Ness, C. G. Sánchez, T. N. Todorov, and A. J. Fisher, Rep. Prog. Phys. 69, 1195 (2006). 